\title{
COVERING COALGEBRAS AND DUAL NON-SINGULARITY
}

\author{
CHRISTIAN LOMP AND VIRGÍNIA RODRIGUES
}

\begin{abstract}
Localisation is an important technique in ring theory and yields the construction of various rings of quotients. Colocalisation in comodule categories has been investigated by some authors where the colocalised coalgebra turned out to be a suitable subcoalgebra. Rather then aiming at a subcoalgebra we look at possible coalgebra covers $\pi: D \rightarrow C$ that could play the rôle of a dual quotient object. Codense covers will dualise dense (or rational) extensions; a maximal codense cover construction for coalgebras with projective covers is proposed. We also look at a dual non-singularity concept for modules which turns out to be the comodule-theoretic property that turns the dual algebra of a coalgebra into a non-singular ring. As a corollary we deduce that hereditary coalgebras and hence path coalgebras are non-singular in the above sense. We also look at coprime coalgebras and Hopf algebras which are non-singular as coalgebras.
\end{abstract}

\section{INTRODUCTION}

Embedding algebras into better ones where certain problems have solutions is one of the major tools in ring theory. An analogous tool for coalgebras does not always exists. Instead of embedding a coalgebra into a better behaved coalgebra one could also try to find a suitable better behaved coalgebra with a projection onto the first one - a covering coalgebra.

The maximal ring of quotients $Q_{\max }(A)$ of an algebra $A$ is such an example of a universal object that has good properties in particular when the algebra in question is non-singular. Recall that an algebra $A$ is called left non-singular if left

2000 Mathematics Subject Classification. 16S90, 16W30.

Key words and phrases. Localisation of coalgebras, Non-singular coalgebras, Hereditary coalgebras, path coalgebras, copolyform modules, maximal ring of quotients.

This work was carried out as part of the project Interacções entre álgebras e co-álgebras between the Universidade do Porto and Universidade Federal de Rio Grande do Sul and Universidade de São Paulo financed through GRICES (Portugal) and CAPES (Brasil) during the second author's visit to the UP. The first author was partially supported by Centro de Matemática da Universidade do Porto (CMUP), financed by FCT (Portugal) through the programs POCTI (Programa Operacional Ciêencia, Tecnologia, Inovação) and POSI (Programa Operacional Sociedade da Informação), with national and European Community structural funds. He would also like to thank the Fundação de Calouste Gulbenkian for travel grant 78123, that allowed him to present this work at the VIII Antalya Algebra Days. The second author was supported by CAPES (Projeto 135/05 - BEX 1378/05-8) and would like to thank the Departamento de Matemática Pura for its hospitality. 
annihilators of non-zero elements are never essential as left ideal. This conditions is a kind of non-commutative torsion-freeness for $A$ and Johnson's theorem states that $A$ is left non-singular if and only if $Q_{\max }(A)$ is von Neumann regular, i.e. the weak global dimension of $Q_{\max }(A)$ is zero.

Throughout the text we will assume that rings $R$ are associative and have a unit. Furthermore we shall write homomorphisms of modules opposite of scalars. A submodule $N$ of a left $R$-module $M$ is called essential (small) if for all proper non-zero $L \subset M: N \cap L \neq 0(N+L \neq M)$. We denote a small submodule $N$ of $M$ by $N \ll M$. Given a module $M$ we denote by $\sigma[M]$ the category of submodules of factor modules of direct sums of copies of $M$ (see [19]). For any pair of modules $X$ and $Y$ we denote the trace of $X$ in $Y$ by $\operatorname{Tr}(X, Y)=\sum\{\operatorname{Im}(f) \mid f \in \operatorname{Hom}(X, Y)\}$.

1.1. The maximal ring of quotients. Given a ring $R$, an overring $S$ of $R$ is called a left ring of quotients if $\operatorname{Hom}_{R-}(S / R, S)=0$. The maximal left ring of quotients $Q_{\max }(R)$ of $R$ is any left ring of quotients such that for any left ring of quotients $S$ of $R$ with embedding $j: R \hookrightarrow S$ there exists a unique ring homomorphism $\varphi: S \rightarrow Q_{\max }(R)$ such that $j \varphi=\imath$ where $\imath: R \hookrightarrow Q_{\max }(R)$ denotes the embedding:

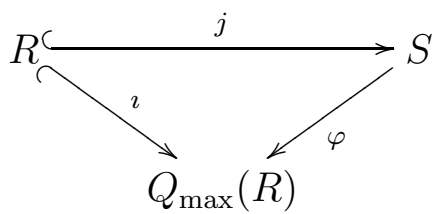

The maximal left ring of quotients exists and can be constructed as follows: Let $E=E(R)$ be the injective hull of $R$ as left $R$-module. Then

$$
Q_{\max }(R):=\{x \in E \mid(x) f=0 \forall f \in \operatorname{End}(E) \text { with }(R) f=0\} .
$$

By construction $Q_{\max }(R)$ is the submodule of $E$ that satisfies $Q_{\max }(R) / R=$ $\operatorname{Re}(E / R, E)$. Where $\operatorname{Re}(X, Y)=\bigcap\{\operatorname{Ke}(f) \mid f: X \rightarrow Y\}$ denotes the reject of $X$ in $Y$.

1.2. Finite dimensional coalgebras. Let us examine the following example: Fix a field $k$ and consider the graph:

$$
a \stackrel{x}{\longrightarrow} b
$$

and the path $k$-coalgebra $C$ associated to this graph which has basis $\{a, x, b\}$ such that $a$ and $b$ are group-like and $\epsilon(x)=0$ with

$$
\Delta(x)=a \otimes x+x \otimes b .
$$

Can we find a coalgebra $D$ and a projection $\pi: D \rightarrow C$ such that $D$ has better properties then $C$ ? First note that $C^{*}$ is isomorphic to the path algebra of the 
given graph and hence

$$
C^{*}=\left(\begin{array}{cc}
k & k \\
0 & k
\end{array}\right) .
$$

The maximal ring of quotients of $C^{*}$ is the full $2 \times 2$-matrix ring $D=M_{2}(k)$ over $k$. Dualizing the inclusion $\imath: C^{*} \rightarrow D$ we get a projection

$$
D^{*} \rightarrow C^{* *} \simeq C
$$

where $D^{*}$ is the $2 \times 2$-matrix coalgebra.

1.3. Analogous to the example above, we can always choose $D$ to be the dual coalgebra of the maximal ring of quotient of a finite dimensional coalgebra in order to obtain a suitable coalgebra cover, as the following theorem shows:

Theorem. Let $C$ be a finite dimensional $k$-coalgebra, then $D=\left(Q_{\max }^{r}\left(C^{*}\right)\right)^{*}$ is a finite dimensional coalgebra and there exists a surjective coalgebra homomorphims $\pi: D \rightarrow C$ whose kernel is small as a right $C$-subcomodule of $D$.

Proof. Since $C$ is finite dimensional, it is a left and right semiperfect coalgebra. Let $P$ be a projective cover of $C$ as right $C$-comodule with epimorphism $\pi: P \rightarrow C$. Since $C$ is finitely generated as left $C^{*}$-module, $P$ is also finitely generated as left $C^{*}$-module and hence finite dimensional. Since $P$ is a projective right $C$-comodule, $P^{*}$ is an injective right $C^{*}$-module (by [2, 9.5]). Moreover as $\pi^{*}: C^{*} \rightarrow P^{*}$ is an essential embedding, $P^{*}$ is isomorphic to the injective hull $E\left(C^{*}\right)$ of $C^{*}$ as right $C^{*}$-module. Since $Q_{\max }^{r}\left(C^{*}\right) \subseteq E\left(C^{*}\right)$, it is also finite dimensional. Hence $D=\left(Q_{\max }^{r}\left(C^{*}\right)\right)^{*}$ is a finite dimensional coalgebra and the transpose $\imath^{*}: D \rightarrow C$ of the algebra embedding $\imath: C^{*} \rightarrow Q_{\max }^{r}\left(C^{*}\right)$ is a surjective coalgebra homomorphism. Since $\imath$ is an essential monomorphism, $\pi$ is a small epimorphism.

1.4. Let $K$ be a field and $\Gamma$ be a quiver, i.e. a directed graph with finitely many vertices $\Gamma_{0}$ and finitely many arrows $\Gamma_{1}$ and without cycles. The path $K$ coalgebra $C$ associated to $\Gamma$ is the vector space whose basis are all paths in $\Gamma$ and with comultiplication $\Delta(w)=\sum_{u v=w} u \otimes v$. For each vertex $i \in \Gamma_{0}$ denote by $v_{i}$ the unique path of length zero at vertex $i$. Note that $C$ is finite dimensional and $C^{*}$ is isomorphic to the path algebra associated to $\Gamma$. Since by [11, 13.25] the right maximal ring of quotients of a right artinian right non-singular ring $A$ is isomorphic $\operatorname{End}\left(\operatorname{Soc}\left(A_{A}\right)\right)$, we only need to determine the right socle of $C^{*}$ to describe the right maximal ring of quotients of $C^{*}$. Let $A$ be the path algebra associated to $\Gamma$. Denote by $\Gamma_{\text {sink }}$ the set of terminal vertices $i \in \Gamma_{0}$, i.e. those vertices from where no arrow starts. Note that for any $i \in \Gamma_{\text {sink }}: v_{i} A=v_{i} K$ is a minimal right ideal of $A$. Moreover for any path $p$ in $A$ which ends at a terminal vertex $i$, the cyclic right ideal $p A$ is a minimal right ideal and isomorpic to $v_{i} A$ since both have the same maximal right ideal $M_{i}$ generated by all paths except $v_{i}$. On the other hand let $I$ be a minimal right ideal of $A$, then $I=\gamma A$ for some 
linear combination $\gamma=\sum_{j=1}^{n} \lambda_{j} p_{j}$ of distinct paths $p_{j}$ and non-zero coeficients $\lambda_{j}$. Let $i^{\prime}$ be the vertex where the path $p_{1}$ ends and choose a path $q$ from $i^{\prime}$ to some terminal vertex $i$. Then $I=\gamma q A$, since $I$ was minimal. Note that $q M_{i}=0$ implies that $I M_{i}=0$, i.e. the annihilator of $I$ is the maximal right ideal $M_{i}$. Hence $I \simeq v_{i} A$. Moreover $\gamma q$ can be written as a linear combination of paths ending at $i$, i.e. $\gamma q=\sum \lambda_{j} p_{j}^{\prime}$ where all paths $p_{j}^{\prime}$ end at $i$. Hence $I \subseteq \bigoplus p_{j}^{\prime} A$. For any terminal vertex $i \in \Gamma_{\text {sink }}$ denote by $P_{i}$ the set of paths ending at $i$ and set $n_{i}=\left|P_{i}\right|$. Then we just showed that

$$
\operatorname{Soc}\left(A_{A}\right)=\bigoplus_{i \in \Gamma_{\text {sink }}}\left(\bigoplus_{p \in P_{i}} p A\right) \simeq \bigoplus_{i \in \Gamma_{\text {sink }}}\left(v_{i} A\right)^{n_{i}}
$$

By [1] the maximal right ring of quotients of $A$ is isomorphic to the endomorphism ring of $\operatorname{Soc}\left(A_{A}\right)$ :

$$
Q_{\text {max }}^{r}(A) \simeq \operatorname{End}\left(\operatorname{Soc}\left(A_{A}\right)\right) \simeq \prod_{i \in \Gamma_{\text {sink }}} \operatorname{End}\left(\left(v_{i} A\right)^{n_{i}}\right) \simeq \prod_{i \in \Gamma_{\text {sink }}} M_{n_{i}}(K),
$$

where $M_{n}(K)$ denotes the ring of $n \times n$-matrizes over $K$.

Going back to our path coalgebra we have now a projection of coalgebras of a direct product of matrix coalgebra onto $C$, i.e.

$$
\prod_{i \in \Gamma_{\text {sink }}} M_{n_{i}}^{c}(K) \rightarrow C .
$$

Here $M_{n}^{c}(K)=\left(M_{n}(K)\right)^{*}$ denotes the $n \times n$-matrix coalgebra with basis $\left\{E_{i j}\right\}_{1 \leq i, j \leq n}$, comultiplication

$$
\Delta\left(E_{i j}\right)=\sum_{l=1}^{n} E_{i l} \otimes E_{l j}
$$

and counit $\epsilon\left(E_{i j}\right)=\delta_{i, j}$.

1.5. In case of an infinite dimensional path coalgebra, how can we obtain a covering coalgebra like the matrix coalgebra in our example ? For instance for the divided power coalgebra, that is the path coalgebra associated to the graph

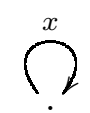

We will see that there is no apropriate coalgebra cover in the sense defined below.

\section{A MODUlE-THEORETIC APPROACH TO COVERING COALGEBRAS}

A module extension $X \hookrightarrow Y$ is called dense if $\operatorname{Hom}(Z / X, Y)=0$ for all $X \subset$ $Z \subset Y$. In [8] Findlay and Lambek proved that the maximal ring of quotient $Q$ of a ring $R$ is the maximal dense extension of $R$ in the category of $R$-modules. 
We will give a module theoretic approach in covering coalgebras using codense covers of modules:

2.1. Codense covers of modules. A module $Y$ is called a cover of $X$ if there exists an epimorphism $\pi: Y \rightarrow X$. The cover $Y$ is said to be small if $\operatorname{Ke}(\pi) \ll Y$ and a cover $Y$ is called a codense cover of $X$ if $\operatorname{Ke}(\pi)$ is a codense submodule of $Y$, that is $\operatorname{Hom}(Y, \operatorname{Ke}(\pi) / L)=0$ for all $L \subseteq \operatorname{Ke}(\pi)$. As a dualisation of dense extensions, codense covers were introduced by Courter in 5. where they are called co-rational extensions. Since the term rational module has a different meaning in the coalgebraic setting, we prefer to refer to 'dense extensions' and 'codense covers' instead. A non-trivial example of a codense cover is the projection $\mathbb{Q} \rightarrow$ $\mathbb{Q} / \mathbb{Z}$, which is codense since $\operatorname{Hom}(\mathbb{Q}, \mathbb{Z} / n \mathbb{Z})=0$ for all $n$.

2.2. Some properties of codense covers can be easily checked:

Lemma. Let $Z$ be a cover of $X$ in $\sigma[M]$.

(1) If $Z$ is a codense cover of $X$, then it is a small cover.

(2) If $Z$ is a small cover of $X$ and $\pi: X \rightarrow Y$ is a codense cover then $\operatorname{Hom}(Z, \operatorname{Ke}(\pi))=0$.

(3) If $Z$ is a projective cover of $X$ in $\sigma[M]$ then a cover $\pi: X \rightarrow Y$ is codense if and only if $\operatorname{Hom}(Z, \operatorname{Ke}(\pi))=0$.

Proof. (1) Let $\pi: Z \rightarrow X$ be a codense cover. Suppose $\operatorname{Ke}(\pi)+Y=Z$, then the canonical projection

$$
Z \rightarrow Z / Y \simeq \operatorname{Ke}(\pi) /(\operatorname{Ke}(\pi) \cap Y)
$$

is zero by hypothesis. Thus $Z=Y$ and $\operatorname{Ke}(\pi) \ll Z$.

(2) Let $p: Z \rightarrow X$ be a small epimorphism and $f \in \operatorname{Hom}(Z, \operatorname{Ke}(\pi))$. Extending $f$ to an homomorphism

$$
g: X=Z / \operatorname{Ke}(p) \rightarrow \operatorname{Ke}(\pi) /(\operatorname{Ke}(p)) f,
$$

mapping $z+\operatorname{Ke}(p) \mapsto(z) f+(\operatorname{Ke}(\pi)) f$, we have $g=0$ since $X$ is a codense cover of $Y$. Thus $(\operatorname{Ke}(p)) f=\operatorname{Im}(f)$. But as $(\operatorname{Ke}(p)) f \ll \operatorname{Im}(f)$, we must have $\operatorname{Im}(f)=0$, i.e. $f=0$.

(3) Since $Z$ is projective cover of $X$ there exists a small epimorphism $p: Z \rightarrow$ $X$. For any $U \subseteq \operatorname{Ke}(\pi)$ and $f: X \rightarrow \operatorname{Ke}(\pi) / U$ we have $p f: Z \rightarrow \operatorname{Ke}(\pi) / U$. Since $Z$ is projective there exists $g: Z \rightarrow \operatorname{Ke}(\pi)$ which is zero by hypothesis. Hence $p f=0$ and $f=0$ as $p$ is an epimorphism. 
2.3. Dual to the definition of a maximal dense extension of a module, we define a maximal codense cover as follows:

Definition 1. Let $X, Y \in \sigma[M]$. A codense cover $p: Y \rightarrow X$ is called a maximal codense cover in $\sigma[M]$ if for any codense cover $\pi: Z \rightarrow X$ there exists a unique epimorphism $\psi: Y \rightarrow Z$ such that $\psi \pi=p$.

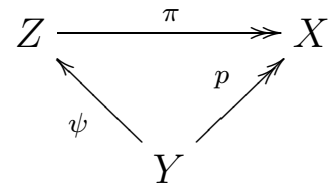

Note that our definition differs from Courter's in [5].

2.4. As it was to expect, in case projective covers exists a dual construction like Findlay and Lambek's allows to construct a maximal codense cover for modules:

Theorem. Let $X \in \sigma[M]$ have a projective cover $P$ in $\sigma[M]$. Denote by $\pi: P \rightarrow$ $X$ the projection and $T:=\operatorname{Tr}(P, \operatorname{Ke}(\pi))$. Then $\widetilde{P}=P / T$ is a maximal codense cover of $X$ in $\sigma[M]$ with induced epimorphism $\widetilde{\pi}: \widetilde{P} \rightarrow X$.

Proof. Note that $\operatorname{Ke}(\tilde{\pi})=\operatorname{Ke}(\pi) / T$ and as $P$ is a projective cover of $\widetilde{P}$, $\operatorname{Hom}(P, \operatorname{Ke}(\pi) / T)=0$. By Lemma 2.2$] \widetilde{\pi}: \widetilde{P} \rightarrow X$ is a codense cover. Let $p: Z \rightarrow X$ be any other codense cover of $X$ in $\sigma[M]$. By the projectivity of $P$ there exist $\psi: P \rightarrow Z$ such that $\psi p=\pi$. As $(T) \psi p=(T) \pi=0$ we deduce

$$
P \operatorname{Hom}(P, \operatorname{Ke}(\pi)) \psi=T \psi \subseteq \operatorname{Ke}(p) .
$$

Since by $\operatorname{Lemma} 2.2 \operatorname{Hom}(P, \operatorname{Ke}(p))=0,(T) \psi=0$. Hence $\psi$ lifts to a homomorphism $\widetilde{\psi}: \widetilde{P} \rightarrow Z$ with $\widetilde{\psi} p=\widetilde{\pi}$.

$\widetilde{\psi}$ is unique because if there existed another map $\phi: \widetilde{P} \rightarrow Z$ with $\phi p=\widetilde{\pi}$, then $\psi-\phi \in \operatorname{Hom}(P, \operatorname{Ke}(p))=0$ by Lemma 2.2 (here we consider $\phi$ as a map from $P$ to $Z$ ).

2.5. For a finite dimensional coalgebra $C$ we saw in 1.3 that $D=\left(Q_{\max }^{r}\left(C^{*}\right)\right)^{*}$ is a small cover of $C$. Actually as it was to expect, $D$ is a maximal codense cover of $C$ in the category of right $C$-comodules:

Theorem. Let $C$ be a finite dimensional coalgebra over a field $k$, then $D=$ $\left(Q_{\text {max }}^{r}\left(C^{*}\right)\right)^{*}$ is a maximal codense cover of $C$ in $\mathcal{M}^{C}$.

Proof. By transposing the embedding $\imath: C^{*} \hookrightarrow Q_{\max }^{r}\left(C^{*}\right)$ we obtained a small cover $\pi: D \rightarrow C$ in 1.3 , where $D=\left(Q_{\max }^{r}\left(C^{*}\right)\right)^{*}$ and $\pi=\imath^{*}$. The kernel $K$ of $\pi$ is isomorphic to $\left(Q_{\max }^{r}\left(C^{*}\right) / C^{*}\right)^{*}$. Note that the dual of any factor comodule $K \rightarrow L$ is a right $C^{*}$-submodule $L^{*}$ of $Q_{\max }^{r}\left(C^{*}\right) / C^{*}$. Hence the transpose map of any right $C$-colinear map $g: C \rightarrow K / L$ yields a right $C^{*}$-linear map $g^{*}:(K / L)^{*} \rightarrow C^{*}$ which could be extended to a right $C^{*}$-linear map from $Q_{\max }^{r}\left(C^{*}\right) / C^{*}$ to $E\left(C^{*}\right)$ and must be zero (where $E\left(C^{*}\right)$ denotes the injective hull 
of $C^{*}$ as right $C^{*}$-module). Hence $D$ is a codense cover of $C$. The maximality follows now by a similar argument, taking into account that any codense cover $D^{\prime}$ of $C$ in $\mathcal{M}^{C}$ would be finitely generated as comodule and hence finite dimensional.

2.6. We will now turn to some examples of modules that are equal its own maximal codense cover. The next Lemma is probably known, but we were unable to find a reference:

Lemma. Every indecomposable non-faithful injective module over a principal ideal domain is uniserial.

Proof. Let $D$ be a principal ideal domain and $M$ an indecomposable nonfaithful injective $D$-module. By Matlis Theorem [14] $M=E(D / \mathfrak{p})$ for some nonzero prime ideal $\mathfrak{p}=D p$ of $D$. Since $D$ is a Dedekind domain, the localisation of $D$ by $\mathfrak{p}: D_{\mathfrak{p}}$ is a discrete valuation ring. Hence $D_{\mathfrak{p}}, Q$ and $Q / D_{\mathfrak{p}}$ are uniserial $D_{\mathfrak{p}}$-modules. Take any $D$-submodule $N \subseteq Q / D_{\mathfrak{p}}$. We will show that $N$ is also a $D_{\mathfrak{p}}$-module. For any $a \notin \mathfrak{p}=D p$ and $n=x / y+D_{\mathfrak{p}} \in N$ with $y=u p^{k} \in \mathfrak{p}$ and $p \nmid u$. Hence $1=r a+s p^{k}$ for some $r, s \in D$. This implies that $\frac{1}{a}-r=\frac{s p^{k}}{a} \in D_{\mathfrak{p}}$. Therefore

$$
\frac{1}{a} n-r n=\frac{s p^{k}}{a} \frac{x}{u p^{k}}=\frac{s x}{a u} \in D_{\mathfrak{p}} \Rightarrow \frac{1}{a} n=r n+D_{\mathfrak{p}} .
$$

Hence the action of $1 / a$ on an element $n$ in $Q / D_{\mathfrak{p}}$ is given by a $D$-scalar multiplication. This shows that $Q / D_{\mathfrak{p}}$ is a uniserial $D$-module. Since $Q / D_{\mathfrak{p}}$ is injective and contains a simple $D_{\mathfrak{p}}$-submodule which is isomorphic to $D_{\mathfrak{p}} / \mathfrak{p} D_{\mathfrak{p}} \simeq D / \mathfrak{p}$, we have that

$$
M \simeq E(D / \mathfrak{p}) \simeq Q / D_{\mathfrak{p}}
$$

is a uniserial $D$-module. Note that all its submodules are of the form $D / \mathfrak{p}^{i}$.

2.7. The next theorem states that indecomposable injectives over suitable rings do not have proper codense covers and as we will see below applies in particular to the case of the divided power coalgebra mentioned in [1.5. A module $M$ is called couniform or hollow if every proper submodule is small.

Theorem. The only possible small covers of a non-faithful indecomposable injective module $M$ over a principal ideal domain $D$ are $M$ and the quotient field $Q$ of $D$.

Proof. By a theorem of Matlis [14, Prop 3.1] $M=E(D / \mathfrak{p})$ for some maximal ideal $\mathfrak{p}$. Furthermore $M$ is uniserial by 2.6. Let $\pi: P \rightarrow M$ be a small cover. Then $P$ is hollow, since $M$ is uniserial and whenever $P=D+E, \pi(D)+\pi(E)=M$, i.e. $\pi(D)=M$ or $\pi(E)=M$ and hence $D=P$ or $E=P$ as $\operatorname{Ke}(\pi) \ll P$.

Since $M$ is injective, $P$ is divisible, because for all $0 \neq x \in D$

$$
\pi(x P)=x \pi(P)=x M=M,
$$


i.e. $x P=P$ as $\pi$ has a small kernel. As $D$ is a principal ideal domain, $P$ is an indecomposable injective $D$-module and again by Matlis theorem $P \simeq Q$ or $P \simeq E(D / \mathfrak{q})$ for some maximal ideal $\mathfrak{q}$. In the later case we must have $\mathfrak{p}=\mathfrak{q}$ since

$$
D / \mathfrak{p}=\operatorname{Soc}(E(D / \mathfrak{p})) \simeq \operatorname{Soc}(E(D / \mathfrak{q}) / \operatorname{Ke}(\pi))=\left(D / \mathfrak{q}^{i+1}\right) /\left(D / \mathfrak{q}^{i}\right) \simeq D / \mathfrak{q}
$$

as $E(D / \mathfrak{q})$ is uniserial and all its submodules are of the form $D / \mathfrak{q}^{i}$.

2.8. The divided power coalgebra is the path coalgebra $C$ associated to the graph

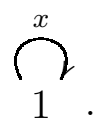

that is the coalgebra over a field $k$ with basis $\left\{1, x, x^{2}, \ldots, x^{i}, \ldots\right\}$ and comultiplication:

$$
\Delta\left(x^{n}\right)=\sum_{i=0}^{n} x^{i} \otimes x^{n-i}
$$

and counit

$$
\epsilon\left(x^{n}\right)=\delta_{0, n} .
$$

Corollary. Let $C$ be the divided power coalgebra over a field $k$. Then $C$ is its own maximal codense cover in the category of $C$-comodules $\mathcal{M}^{C}$.

Proof. The dual algebra $C^{*} \simeq k[[Z]]$ of $C$ is the power series ring in one variable, by the isomorphism:

$$
f \mapsto \sum_{n=0}^{\infty} f\left(x^{n}\right) Z^{n}
$$

Note that the power series ring in one variable is a discrete valuation ring, e.g. a principal ideal domain. Since $C$ is an injective cogenerator in $\mathcal{M}^{C}$ with simple coradical $C_{0}=k 1, C$ is a non-faithful indecomposable injective $C^{*}$-module over the discrete valuation ring $C^{*}$. By Theorem 2.7 the only small covers of $C$ in $C^{*}$-Mod are $C$ and the quotient field $Q$ of $C^{*}$. Since $C^{*}$ is not a $C$-comodule, $Q$ is also not a $C$-comodule. Hence the only small cover of $C$ as $C$-comodule is $C$ itself.

\section{DUAL NON-SINGUlarity OF MODUleS}

Recall that a left $R$-module $M$ is called singular if every element of $M$ is annihilated by an essential left ideal of $R$. An $R$-module $M$ is called non-singular if it contains no non-zero singular submodule. 
3.1. Non-singularity generalises torsion-freeness of modules to the non-commutative setting. Lambek's torsion theory is the right concept for a module theoretic setting in which the construction of maximal dense extension of modules are put. Dual Goldie torsion theories have been studied by various authors [17, [9, [13]. As singular modules play the rôle of torsion modules, small modules will play a similar rôle in the dual situation. Let $\mathcal{S}$ be the class of small modules in $\sigma[M]$, i.e. those which are small in their injective hull in $\sigma[M]$. $\mathcal{S}$ is a Serre class, i.e. it is closed under submodules, factor modules and extensions (and hence also under finite direct sums). Define

$$
\rho(X)=\operatorname{Re}(X, \mathcal{S})=\bigcap\{U \subseteq X \mid X / U \in \mathcal{S}\}
$$

for any $X \in \sigma[M]$ and call $X$ dual non-M-singular if $\rho(X)=X$. These are precisely those modules which do not have any non-zero small homomorphic image.

Since an injective module is a direct summand in any extension, injectives are never small. Hence cohereditary modules, i.e. those all whose factor modules are injective, are examples of dual non- $M$-singular modules. On the other hand there exist examples of injective modules that are subdirect products of their $M$-small factor modules (see Zoeschinger [22]).

3.2. Pushing singularity to smaller categories like $\sigma[M]$ needed a characterisation that was free of refeering to left ideals of a ring. Concepts for Singularity and their duals had been already proposed in some abelian categories by Pareigis [16] and it is not difficult to see that in the module case a module $M$ is singular if and only if it is a factor module of a module by an essential submodule. In the case of $\sigma[M]$ it turned out, as shown in [20, that non-singularity of $M$ could be characterised by the internal property that any essential submodule is dense. This property has been studied by Zelmanowitz in [21] where he also termed it polyform. It is not difficult to dualise those notions, but it turns out that they are not always equivalent.

3.3. Dual to a polyform module, call a module $M$ copolyform if for every small submodule $K$ of $M$, the canonical projection $M \rightarrow M / K$ is a codense cover. Note that dual non- $M$-singular modules $X$ in $\sigma[M]$ are copolyform since for any small submodule $K$ of $X$ any factor module $K / L$ is also $M$-small and thus $\operatorname{Hom}(X, K / L)=0$, i.e. $X \rightarrow X / K$ is codense. The converse is not true, e.g. $\mathbb{Z}$ is copolyform, but not non- $\mathbb{Z}$-small. Copolyform modules had been introduced in [12] and were studied also in [18].

3.4. By definition it is clear that copolyform modules can be characterised by their homomorphisms to factor modules. For any two modules $X$ and $Y$ set

$$
\nabla(X, Y)=\{f \in \underset{9}{\operatorname{Hom}}(X, Y) \mid \operatorname{Im}(f) \ll Y\} .
$$


This set has been introduced by Beidar and Kasch in [1] were it was termed the cosingular ideal of $X$ and $Y$. Suppose $M$ is copolyform and $f \in \nabla(M, M / N)$ for some $N \ll M$ then $\operatorname{Im}(f)=K / N \ll M / N$ and $N \ll M$ implies $K \ll M$. But as the projection $M \rightarrow M / K$ is codense, $f \in \operatorname{Hom}(M, K / N)=0$. Thus $\nabla(M, M / N)=0$. On the contrary, if $\nabla(M, M / N)=0$ for all $N \ll M$ then for any small cover $\pi: M \rightarrow F$ with $K=\operatorname{Ke} \pi \ll M$ and submodule $L \subseteq K$ we have $\operatorname{Hom}(M, K / L) \subseteq \nabla(M, M / L)=0$. Hence $\pi: M \rightarrow F$ is a codense cover. We have just proved the following statement:

Theorem. An R-module $M$ is copolyform if and only if $\nabla(M, M / N)=0$ for all $N \ll M$.

Choosing $N=0$ in the above Theorem, we get that a copolyform module has no non-zero homomorphism with small image, i.e. $\nabla(M):=\nabla(M, M)=0$. Note that under some suitable projectivity conditions $\nabla(M)$ equals Jac $(\operatorname{End}(M))$.

3.5. Note that for self-projective modules $M, \nabla(M)=\operatorname{Jac}(\operatorname{End}(M))$ (see [19]).

Theorem. A self-projective module $M$ is copolyform if and only if $\operatorname{Jac}(\operatorname{End}(M))=$ 0 .

Thus a ring $R$ is copolyform as left $R$-module if and only if it is semiprimitive.

3.6. Since our aim is to apply the module theoretic terms above to the situation of coalgebras, recall that any coalgebra $C$ of a field $k$ is an injective cogenerator in the category $\mathcal{M}^{C}$ of right $C$-comodules. Moreover there exists an antiisomorphism of rings between the dual algebra $C^{*}$ and the endomorphism of $C$ as right $C$-comodule and an isomorphism of rings between $C^{*}$ and the endomorphism of $C$ as left $C$-comodule:

$$
\operatorname{End}\left({ }_{C^{*}} C\right)^{o p} \simeq C^{*} \simeq \operatorname{End}\left(C_{C^{*}}\right)
$$

Under some light injectivity and cogenerator properties we can say much more about copolyform modules. A module $Q$ is called pseudo-injective with respect to a non-zero monomorphism $f: Y \hookrightarrow X$ if for all non-zero $g: Y \rightarrow Q$ there exist $h \in \operatorname{End}(Q)$ and $k \in \operatorname{Hom}(X, Q)$ such that $f k=g h \neq 0$. A module $Q$ is called pseudo-injective in $\sigma[M]$ if it is pseudo-injective with respect to all non-zero monomorphism $f: Y \hookrightarrow X$ in $\sigma[M]$.

Lemma. Let $M$ be pseudo-injective in $\sigma[M]$. Then $\operatorname{Hom}(M / N, M)=0$ for all submodules $N$ such that $M / N$ is $M$-small provided $\nabla(M)=0$.

Proof. Assume that $M / N$ is small in some module $X \in \sigma[M]$ and let $f: M / N \rightarrow M$ be a homomorphism. Suppose $f$ is non-zero then by pseudoinjectivity there are homomorphisms $h \in \operatorname{End}(M)$ and $k \in \operatorname{Hom}(X, M)$ such that $f h=i k \neq 0$ where $i: M / N \hookrightarrow X$ denotes the inclusion. Since homomorphic images of small modules are small, $\operatorname{Im}(f h)=\operatorname{Im}(i k) \ll M$. Considering the projection $p: M \rightarrow M / N$ we get a homomorphism $p f h \in \operatorname{End}(M)$ whose image 
is small in $M$. Since $\nabla(M)=0, p f h=0$ which implies $f h=0$, a contradiction. Thus $\operatorname{Hom}(M / N, M)=0$.

3.7. Lemma 3.6] shows that a pseudo-injective module $M$ with $\operatorname{Hom}(M / N, M) \neq$ 0 for all non-zero $N \subseteq M$, is dual non- $M$-singular if and only if $\nabla(M)=$ 0 . We will show that this is also equivalent to $\operatorname{End}(M)$ being non-singular. Say that a module $M$ is coretractable if for all non-zero submodules $N$ of $M$ : $\operatorname{Hom}(M / N, M) \neq 0$. We first need the following Lemma

Lemma. Let $M$ and $Q$ be left $R$-modules and $T:=\operatorname{End}(Q)$. Denote by $Z\left(M^{*}\right)$ the singular submodule of $M^{*}:=\operatorname{Hom}(M, Q)$ as right $T$-module. Suppose that $Q$ is coretractable then

$$
Z\left(M^{*}\right) \subseteq \nabla(M, Q)
$$

holds. If moreover $Q$ is pseudo-injective with respect to all monomorphisms of the form $g: Q / \operatorname{Ke} g \hookrightarrow Q$ for any $0 \neq g \in T$ then equality hold, i.e. $Z\left(M^{*}\right)=$ $\nabla(M, Q)$.

Proof. Take $f \in Z\left(M^{*}\right)$. Then $A n n_{T}(f)=\{g \in T \mid f g=0\}$ is essential in $T$. Suppose $\operatorname{Im}(f)+U=Q$ for some submodule $U$ of $Q$. Then $A n n_{T}(f) \cap A n n_{T}(U)=$ $A n n_{T}(\operatorname{Im}(f)+U)=0$ implies $\operatorname{Hom}(Q / U, Q)=A n n_{T}(U)=0$. By hypothesis $U=Q$, i.e. $\operatorname{Im}(f) \ll Q$ and $f \in \nabla(M, Q)$.

Now assume that $Q$ is pseudo-injective with respect to all monomorphisms $g: Q / \operatorname{Ke}(g) \hookrightarrow Q$. Let $f \in \nabla(M, Q)$ and $g \in T$ such that $g T \cap A n n_{T}(f)=0$. Suppose there exists a non-zero $h \in A n n_{T}(\operatorname{Ke} g) \cap A n n_{T}(\operatorname{Im}(f))$. As $h$ defines a non-zero homomorphism from $Q / \operatorname{Ke} g$ to $Q$ we have by hypothesis endomorphisms $k, l \in T$ such that $0 \neq g k=h l$. But as $h \in A n n_{T}(\operatorname{Ke} g) \cap A n n_{T}(f)$, we have $h l=g k \in g T \cap A n n_{T}(f)=0$; a contradiction. Thus $A n n_{T}(\operatorname{Ke} g) \cap$ $A n n_{T}(\operatorname{Im}(f))=0$ and

$0=A n n_{T}(\operatorname{Ke} g) \cap A n n_{T}(\operatorname{Im}(f))=A n n_{T}(\operatorname{Ke} g+\operatorname{Im}(f)) \simeq \operatorname{Hom}(Q /(\operatorname{Ke} g+\operatorname{Im}(f)), Q)$.

Since $Q$ is coretractable, $\operatorname{Ke} g+\operatorname{Im}(f)=Q$, but as $\operatorname{Im}(f) \ll Q, g=0$.

Note that the condition in Lemma 3.7(2) is fulfilled if $Q$ is semi-injective, i.e. injective with respect to all monomorphisms of the above form, or if $Q$ is pseudoinjective in $\sigma[Q]$.

3.8. The last Lemma 3.7 together with 3.6 enables us to characterise those copolyform modules which are injective cogenerators:

Theorem. Let $M$ be a coretractable left $R$-module that is pseudo-injective in $\sigma[M]$. Then the following statements are equivalent:

(a) $M$ is dual non-singular in $\sigma[M]$.

(b) $M$ is copolyform.

(c) $\nabla(M)=0$.

(d) $\operatorname{End}(M)$ is a right non-singular ring. 
3.9. The lattice of submodules of a module is pseudo-complemented, but its dual lattice does not need to be. To overcome this problem while dualising module theoretic notions, one has to make suitable assumption on the lattice of submodules. An $R$-module $M$ is called weakly supplemented if any submodule $N$ of $M$ has a weak supplement, that is a submodule $L$ of $M$ such that $N+L=M$ and $N \cap L \ll M$. This is a weak form of a pseudo-complement in the dual lattice of submodules of $M$.

Theorem. The following statements are equivalent for a weakly supplemented module:

(a) $M$ is copolyform.

(b) $\nabla(M, M / N)=0$ for all $N \subseteq M$.

(c) Every factor module of $M$ is copolyform.

(d) $\nabla(M)=0$ and $M$ is $M$-im-small-projective, i.e. any diagram

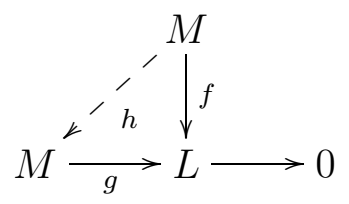

with $\operatorname{Im}(f) \ll L$ can be commutatively extended by some $h: M \rightarrow M$.

Proof. $\quad(a) \Rightarrow(b)$ Let $f: M \rightarrow M / N$ have small image and choose a weak supplement $L$ of $N$. Thus $M / N=(N+L) / N \simeq L /(L \cap N) \subseteq M /(L \cap N)$. Since $L \cap N \ll M$ and $f \in \nabla(M, M /(L \cap N)$, we have $f=0$ by $(a)$.

$(b) \Rightarrow(c)$ let $N \subseteq L \subseteq M$ such that $L / N \ll M / N$ and $f \in \nabla(M / N, M / L)$. Then $f \pi_{N} \in \nabla(M, M / L)=0$, i.e. $f=0$. Hence $M / N$ is copolyform.

$(c) \Rightarrow(a)$ is trivial and $(b) \Rightarrow(d)$ is clear, since for $N=0, \nabla(M, M)=$ $\nabla(M)=0$ and as $\nabla(M, M / N)=0$ for all factor modules $L$ of $M$, there are no non-zero homomorphisms $f: M \rightarrow L$ with small image, i.e. $M$ is trivially $M$-im-small projective.

$(d) \Rightarrow(a)$ Let $f \in \nabla(M, M / N)$ with $N \ll M$ and denote by $\pi_{N}: M \rightarrow M / N$ the canonical projection. By $M$-im-projectivity there exists $h: M \rightarrow M$ such that $\pi_{N} h=f$. Since $\operatorname{Im}(f)=\operatorname{Im}\left(\pi_{N} h\right) \ll M / N$ and $N \ll M$, we have $\operatorname{Im}(h) \ll M$, i.e. $h \in \nabla(M)=0$. Thus $f=0$.

A module which satisfies condition $(c)$ is also called strongly copolyform. This is in general a stronger condition then copolyformness. In [18] strongly copolyform modules are called copolyform.

3.10. A module $M$ is called couniform or hollow if $N+L=M$ implies $N=$ $M$ or $L=M$ for all proper submodules $N, L$ of $M$. Uniserial modules are couniform and couniform modules are indecomposable. Furthermore couniform modules are trivially weakly supplemented since all proper submodules are small. From the last characterisation of copolyform modules we easily deduce that a couniform module is copolyform if and only if every projection $M \rightarrow M / N$ for 
any proper submodule $N$ of $M$ is codense. Couniform copolyform modules are called epiform and satisfy the property that all of their non-zero endomorphisms are epimorphisms. The converse holds under some suitable assumptions as we will see later.

3.11. In case of couniform modules we deduce from 3.8 the following

Corollary. Let $M$ be a couniform coretractable left $R$-module that is pseudoinjective in $\sigma[M]$. Then the following statements are equivalent:

(a) $M$ is dual non-M-singular.

(b) $M$ is epiform.

(c) Every non-zero endomorphism of $M$ is an epimorphism.

(d) Every non-zero homomorphism from a factor module $L$ of $M$ to $M$ is surjective.

(e) $\operatorname{End}(M)$ is a domain.

Proof. $(a) \Leftrightarrow(b)$ follows from 3.8 .

$(b) \Rightarrow(c)$ For any $0 \neq f \in \operatorname{End}(M), \operatorname{Im}(f) \nless M$ as $\nabla(M)=0$. Thus $\operatorname{Im}(f)=$ $M$.

$(c) \Rightarrow(d)$ Let $f: M / N \rightarrow M$ since $\pi_{N} f$ is an epimorphism of $M, f$ has to be an epimorphism (here $\pi_{N}$ denotes the projection).

$(d) \Rightarrow(e)$ If $f g=0$, then $\operatorname{Im}(f) \subseteq \operatorname{Ke}(g)$. And if $f \neq 0$, then $M=\operatorname{Im}(f)=$ $\operatorname{Ke}(g)$, i.e. $g=0$.

$(e) \Rightarrow(a)$ follows from 3.8 as domains are non-singular.

3.12. Copolyform module with projective covers can be characterise by their endomorphism rings.

Proposition. Let $M$ be an $R$-module with projective cover $P$ in $\sigma[M]$. Then $M$ is copolyform if and only if $\operatorname{Jac}(\operatorname{End}(P))=0$.

Proof. Recall that $\operatorname{Jac}(\operatorname{End}(P))=\nabla(P)$. Assume $M$ to be copolyform and let $f \in \nabla(P)$. Then, for any $g \in \operatorname{Hom}(P, M), U:=\operatorname{Im}(f g) \ll M$. However, by Lemma 2.2, $\operatorname{Hom}(P, U)=0$ and so $f g=0$. This implies $\operatorname{Im}(f) \subseteq \operatorname{Ke}(g)$ and so

$$
\operatorname{Im}(f) \subseteq \bigcap\{\operatorname{Ke}(g): g \in \operatorname{Hom}(P, M)\}=\operatorname{Re}(P, M)=0,
$$

as $P$ is cogenerated by $M$ (see [19, 18.4]). Thus $f=0$, i.e. $\operatorname{Jac}(\operatorname{End}(P))=0$. On the contrary if $\nabla(P)=0$, then $P$ is copolyform by 3.5. Denote by $p: P \rightarrow M$ the projection and let $\pi: M \rightarrow X$ be any small cover. The composition $p \pi: P \rightarrow$ $X$ is also a small cover and therefore codense. In particular $\operatorname{Hom}(P, \operatorname{Ke}(p \pi))=0$ and, by projectivity of $P, \operatorname{Hom}(P, \operatorname{Ke}(\pi))=0$. By $2.2 \pi$ is a codense cover, i.e. $M$ is copolyform. 
3.13. The last proposition showed that a projective cover of a copolyform module is copolyform as well.

Corollary. Let $M$ be a copolyform module with projective cover $P$ in $\sigma[M]$, then $\operatorname{End}(M)$ is a subring of $\operatorname{End}(P)$ such that every epimorphism $f \in \operatorname{End}(M)$ with small kernel is invertible in $\operatorname{End}(P)$.

Proof. Denote by $p: P \rightarrow M$ the projection and take any non-zero $f \in$ $\operatorname{End}(M)$. Then by the projectivity of $P$, there exists a non-zero $\bar{f} \in \operatorname{End}(P)$ such that $p f=\bar{f} p$. Suppose there exists another $g \in \operatorname{End}(P)$ such that $p f=g p$, then

$$
0=p f-p f=(\bar{f}-g) p
$$

implies $\operatorname{Im}(\bar{f}-g) \subseteq \operatorname{Ke}(p)$, i.e. $\bar{f}-g \in \nabla(P)=0$. Hence $\bar{f}=g$. Thus the correspondence $f \mapsto \bar{f}$ is uniquely defined.

Now assume that $f$ is an epimorphism with small kernel, then $p f=\bar{f} p$ implies that $\bar{f} p$, and hence $\bar{f}$ is an epimorphism with small kernel. By the projectivity of $P, \bar{f}$ splits and, as $\operatorname{Ke}(\bar{f}) \ll P$, must be an isomorphism.

3.14. The existence of a projective cover, turns the class $\mathcal{S}$ of $M$-small modules into a cotorsion class:

Proposition. Assume that $M$ is dual non-singular in $\sigma[M]$ and has a projective cover $P$ in $\sigma[M]$. Then the class of small modules in $\sigma[M]$ is closed under submodules, factor modules, extensions and direct products (in $\sigma[M]$ ) and can be described as:

$$
\mathcal{S}=\{X \in \sigma[M]: \operatorname{Hom}(P, X)=0\}
$$

Moreover for any $Z \in \sigma[M], \rho(Z)=\operatorname{Re}(Z, \mathcal{S})$ is dual non-M-singular and $Z / \rho(Z)$ is $M$-small.

Proof. Note that if $\mathcal{S}$ can be described as stated above, then it also satisfies the closure properties. Hence we only need to show that $\mathcal{S}$ equals the class of modules $X$ with $\operatorname{Hom}(P, X)=0$. Let $X$ be any module in $\sigma[M]$ and $\widehat{X}$ its injective hull in $\sigma[M]$. By [19, 17.9], $\widehat{X}$ is $M$-generated and hence $P$-generated. If $X$ is not $M$-small, then it is not small in its $M$-injective hull $\widehat{X}$. Thus assume there is a proper submodule $Y$ of $\widehat{X}$ such that $X+Y=\widehat{X}$. Then $X /(X \cap Y) \simeq$ $\widehat{X} / Y$ is a nonzero $P$-generated $R$-module. Hence there is an index set $\Lambda$ and an epimorphism $f: P^{(\Lambda)} \rightarrow X /(X \cap Y)$ and so, since $P^{(\Lambda)}$ is projective in $\sigma[M]$, $f$ can be lifted to a homomorphism $g: P^{(\Lambda)} \rightarrow X$, i.e. $\operatorname{Hom}(P, X) \neq 0$. Hence $X \notin \mathcal{S} \Rightarrow \operatorname{Hom}(P, X) \neq 0$.

On the other hand assume $0 \neq X \in \mathcal{S}$ and $f \in \operatorname{Hom}(P, X)$. Denote by $Y=$ $\operatorname{Im}(f)$ and let $\pi: P \rightarrow M$ be the projection. Then extend $f$ to a homomorphism

$$
g: M \simeq P / \operatorname{Ke}(\pi) \rightarrow Y /(\operatorname{Ke}(\pi)) f
$$


sending $p+\operatorname{Ke}(\pi)$ to $(p) f+(\operatorname{Ke}(\pi)) f$. Since $M$ is dual non-M-singular, $g=0$ and $Y=\operatorname{Im}(f) \subseteq(\operatorname{Ke}(\pi)) f$. Thus $P=\operatorname{Ke}(\pi)+\operatorname{Ke}(f)$, but since $\operatorname{Ke}(\pi) \ll P$, $\operatorname{Ke}(f)=P$ and $f=0$. This shows that $X \in \mathcal{S}$ implies $\operatorname{Hom}(P, X)=0$ proving the equality of the classes indicated.

Thus $\mathcal{S}$ is closed under submodules, factor modules, direct products and extensions. Note that it follows also that $P$ is dual non- $M$-singular. Moreover since $Z / \rho(Z)$ is a subdirect product of $M$-small modules, it is $M$-small. Furthermore, since $P$ is projective and

$$
\operatorname{Hom}(P, \rho(Z) / \operatorname{Tr}(P, \rho(Z)))=0,
$$

we must have $\rho(Z)=\operatorname{Tr}(P, \rho(Z))$, i.e. $\rho(Z)$ is $P$-generated and therefore dual non- $M$-singular.

In the case above, $P$ generates the cotorsion theory whose cotorsion modules are the $M$-small modules in $\sigma[M]$. the cotorsion free modules are precisely the $P$-generated modules.

\section{Non-Singular CoAlgebras}

Having defined a dual non-singularity concept for modules, we are going to apply it to comodules. Let $C$ be a coalgebra over a field $k$. Any right $C$-comodule $M$ carries a natural left $C^{*}$-module structure. Call a right(left) $C$-comodule $M$ copolyform (resp. epiform) if it is copolyform (resp. epiform) as left(right) $C^{*}$ module.

Theorem 3.8 and the facts on coalgebras 3.6 yield the following

Theorem. Let $C$ be a coalgebra over a field $k$. Then the following statements are equivalent:

(a) $C$ is a copolyform right $C$-comodule.

(b) $\operatorname{End}\left({ }_{C^{*}} C\right)$ is a right non-singular ring.

(c) $C^{*}$ is a left non-singular ring.

(d) $\operatorname{End}\left(C_{C^{*}}\right)$ is a left non-singular ring.

(e) $C$ is a copolyform left $C$-comodule.

Any coalgebra that satisfies one of the above conditions is called non-singular.

Proof. $(a) \Leftrightarrow(b)$ follows from Theorem 3.8 .

$(b) \Leftrightarrow(c)$ follows from the anti-isomorphism between $\operatorname{End}\left({ }_{C^{*}} C\right)$ and $C^{*}$.

$(c) \Leftrightarrow(d)$ follows from the isomorphism between $\operatorname{End}\left(C_{C^{*}}\right)$ and $C^{*}$.

$(d) \Leftrightarrow(e)$ follows from Theorem 3.8 (for right $R$-modules).

4.1. In [15], Nastasescu, Torrecillas and Zhang called a coalgebra $C$ hereditary if $C$ is a cohereditary left (and/or right) $C$-comodule. By our remark in 3.1 cohereditary modules are dual non-singular. Hence by 3.8 any hereditary coalgebra is non-singular. Chin showed in [3] that any path coalgbera is hereditary. 
Furthermore Chin and Montgomery showed in 4] that any coalgebra over an algebraically closed field is Morita-Takeuchi equivalent to a subcoalgebra of a path coalgebra. Thus hereditary and hence non-singular coalgebras are ubiquitous.

4.2. In [15] it has been also proven that a finite dimensional coalgebra $C$ is hereditary if and only if $C^{*}$ is left hereditary. Since there are finite dimensional algebras which are left non-singular, but not left hereditary, we can construct coalgebras which are non-singular but not hereditary. Let $k$ be a field let $R$ be any finite dimensional $k$-algebra which is not left hereditary; for example $R=k[x] /\left(x^{2}\right)$. Then define

$$
A=\left(\begin{array}{ll}
k & 0 \\
R & R
\end{array}\right)
$$

By [10, 4.4.3], $A$ is right non-singular, but not right hereditary by [10, 4.4.7] as $R$ is not right hereditary. Hence $C=\left(A^{o p}\right)^{*}$ is a non-singular coalgebra which is not hereditary.

4.3. Call a coalgebra $C$ cosemiprime if $I \wedge I \neq C$ holds for all proper subcoalgebras $I$ of $C$. It is not difficult to see that $C$ is a cosemiprime coalgebra if and only if $C^{*}$ is semiprime and we deduce that a cocommutative coalgebra is non-singular if and only if $C$ is cosemiprime.

\subsection{The strict hierarchie of coalgebraic properties}

$$
\text { cosemisimple } \Rightarrow \text { hereditary } \Rightarrow \text { non-singular }
$$

collapses when assuming some flatness condition on the coalgebra: Since a coalgebra $C$ is flat as right $C^{*}$-module if and only if $C^{*}$ is left self-injective (see [2]), we have that the dual algebra $C^{*}$ of a non-singular coalgebra $C$ which is flat as left $C^{*}$-module must be a left self-injective and left non-singular ring and hence von Neumann regular (as it equals its own maximal left ring of quotient). Note that a von Neumann regular ring is semiprimitive, hence $\operatorname{Jac}\left(C^{*}\right)=0$. By [2, $\operatorname{Jac}\left(C^{*}\right)=C_{0}^{\perp}$ where $C_{0}$ denotes the coradical of $C$. Hence $C_{0}^{\perp}=0$ implies $C=C_{0}$. We just proved the following theorem:

Theorem. A coalgebra $C$ is cosemisimple if and only if $C$ is non-singular and flat as right $C^{*}$-module.

Since finite dimensional Hopf algebras are projective as comodule, we deduce that finite dimensional Hopf algebra which are right non-singular coalgebras are cosemismple.

4.5. The characterisation 3.11 of epiform modules yields that a coalgebra $C$ is epiform as right (or left) comodule if and only if $C^{*}$ is a domain. Recall that a coalgebra $C$ is called coprime if $C^{*}$ is a prime ring. As we see, any coalgebra that is epiform as coalgebra is a coprime coalgebra. In case $C$ is cocommutative those notions are equivalent. 
4.6. We are going to show that there exists a dichotomie for coprime coalgebras that states that over a coprime coalgebra either every comodule is projective or no non-trivial comodule is projective.

Theorem. The following statements are equivalent for a coprime coalgebra $C$ over a field $k$ :

(a) $C$ is a matrix $k$-coalgebra, i.e. $C^{*}$ is a matrix algebra over a division ring.

(b) $C^{*}$ is a simple ring.

(c) $C$ is finite dimensional.

(d) Every non-zero right (left) $C$-comodule is projective as $C^{*}$-module.

(e) There exists a non-zero projective right (left) $C$-comodule.

(f) No non-zero right (left) $C$-comodule is singular as $C^{*}$-module.

(g) There exists a non-zero right (left) $C$-comodule that is not singular as a $C^{*}$-module.

(h) Every right (left) $C$-comodule is injective.

Proof. $\quad(a) \Rightarrow(b)$ is clear.

(b) $\Rightarrow\left(\right.$ c) assume $C^{*}$ is simple, then $C$ is a simple coalgebra, because if $D$ is any subcoalgebra of $C$, then $D^{\perp}$ is an ideal of $C^{*}$ and hence 0 or $C^{*}$,i.e. $D=C$ or $D=0$. Since any non-zero element of $C$ is contained in a non-zero finite dimensional subcoalgebra of $C, C$ must be finite dimensional.

(c) $\Rightarrow$ (a) since $C$ is finite dimensional, $C^{*}$ is finite dimensional. As $C^{*}$ is also a prime ring, it must be a matrix algebra.

(a) $\Rightarrow(\mathrm{d})$ is clear.

$(\mathrm{d}) \Rightarrow(\mathrm{e}) \Rightarrow(\mathrm{g})$ and $(\mathrm{d}) \Rightarrow(\mathrm{f}) \Rightarrow(\mathrm{g})$ are trivial since projective modules are not singular.

$(\mathrm{g}) \Rightarrow(\mathrm{c})$ Suppose $M$ is a non-zero left $C$-comodule which is not singular as $C^{*}$-module. Then there exists a $C^{*}$-submodule $N$ of $M$ which is not singular. We might choose $N$ to be a cyclic $C^{*}$-submodule of $M$. Since comodules are locally finite dimensional, $N$ is finite dimensional. As the annihilator $\operatorname{Ann}_{C^{*}}(N)$ is not an essential left ideal of $C^{*}$, but all non-zero ideals of a prime ring are essential as left ideals, we conclude that $\operatorname{Ann}_{C^{*}}(N)=0$, thus

$$
C^{*}=C^{*} / \operatorname{Ann}_{C^{*}}(N) \hookrightarrow \oplus_{i=1}^{s} C^{*} / \operatorname{Ann}_{C^{*}}\left(n_{i}\right)
$$

is finite dimensional, where $n_{i}$ is a generating set of $N$.

$(a) \Leftrightarrow(h)$ is clear, since $C$ is cosemisimple.

4.7. By negating $(c),(e)$ and $(g)$ we get of the last Theorem we deduce the following

Corollary. The following statements are equivalent for a coprime coalgebra $C$ over a field $k$.

(a) C has infinite dimension. 
(b) Every right or left $C$-comodule is singular as $C^{*}$-module.

(c) There is no non-zero projective object in the category of right or left $C$ comodules.

4.8. The last Corollary shows the dichotomie of coprime coalgebras: Either every comodule is coalgebra and the coalgebra is necessarily a matrix coalgebra or every comodule is singular as $C^{*}$-module and $\mathcal{M}^{C}$ has no non-zero projective object.

This dichotomie shows also that we can not use projective cover to build maximal codense covers of infinite dimensional coprime coalgebras.

4.9. From 3.11 have that any $C$ which is epiform is either the dual of a finite dimensional divison algebra $K$ over $k$ or infinite dimensional such that the category of right $C$-comodules consists of torsion $C^{*}$-modules, in particular there are no non-zero projective objects in $\mathcal{M}^{C}$.

4.10. Note that any coalgebra $C$ can be written as a sum of indecomposable injective comodules $E_{\lambda}$. If $C$ is cocommutative then each of the $E_{\lambda}$ is actually a subcoalgebra of $C$. Assume now that $C$ is a cocommutative semiperfect coalgebra over a field $k$, then $C=\bigoplus_{\lambda} E_{\lambda}$ is a direct coproduct of finite dimensional cocommutative indecomposable coalgebras. If moreover $C$ is non-singular, then each of the $E_{\lambda}$ is also non-singular and $E_{\lambda}^{*}$ is a finite dimensional commutative semiprime $k$-algebra. Thus $E_{\lambda}^{*}$ is a finite field extension $K_{\lambda}$ of $k$ and $E_{\lambda}=K_{\lambda}^{*}$ is a finite dimensional simple coalgebra. Thus we have proved the following

Theorem. Any cocommutative non-singular and semiperfect coalgebra is cosemisimple.

\section{REFERENCES}

[1] Beidar, K. and Kasch, F., Good conditions for the total. in "International Symposium on ring theory", Birkenmeier, Gary F. (ed.) et all, Birkäuser, Trends in Mathematics 43-65 (2001)

[2] Brzezinski, T. and Wisbauer, R., Corings and Coalgebras, LMS Lecture Notes Series 309 (2003)

[3] Chin, W, Hereditary and path coalgebras., Commun. Algebra 30(4), 1829-1831 (2002)

[4] Chin, W. and Montgomery, S., Basic coalgebras., in "Modular interfaces. Modular Lie algebras, quantum groups, and Lie superalgebras." ed. Chari, V. et al., AMS/IP Stud. Adv. Math. 4, 41-47 (1997)

[5] Courter, R.C., The maximal co-rational extension by a module, Can. J. Math. 18, 953-962 (1966).

[6] Cuadra, J. and Gómez-Torrecillas, J., Serial coalgebras., J. Pure Appl. Algebra 189(1-3), 89-107 (2004)

[7] Cuadra, J. and Gómez-Torrecillas, J., Idempotents and Morita-Takeuchi theory., Commun. Algebra 30(5), 2405-2426 (2002)

[8] Findlay, G.D. and Lambek, J., A generalized ring of quotients. I, II., Can. Math. Bull. 1, 77-85, 155-166 (1958). 
[9] Generalov,A.I., A radical dual to Goldie torsion., Abelevy Grupy Moduli 11-12, 70-77 (1994)

[10] Goodearl,K.R., Ring Theory Nonsingular Rings and Modules, Monographs and Textbooks in Pure and Applied Mathematics, (1976)

[11] Lam, T.Y., Lectures on modules and rings, Graduate texts in Mathematics 189, Springer (1999)

[12] Lomp, C., On dual Goldie dimension, MSc Thesis, Univ. Glasgow (1997)

[13] Lomp, C., The splitting of the dual Goldie torsion theory, Contemporary Math. (2001)

[14] Matlis, E., Injective modules over noetherian rings., Pac. J. Math. 8, no. 3, 511-528 (1958)

[15] Nastasescu, C., Torrecillas, B. and Zhang, Y.H., Hereditary coalgebras., Commun. Algebra 24(4), 1521-1528 (1996)

[16] B.Pareigis, Radikale und kleine Moduln (Radicals and small modules), Bayer. Akad. Wiss., Math.-naturw. Kl., Sitzungsber. 1965, 185-199, 1966.

[17] Ramamurthi,V.S., The smallest left exact radical containing the Jacobson radical., Ann. Soc. Sci. Bruxelles Ser. I 96(4), 201- 206 (1982)

[18] Talebi, Y. and Vanaja, N., Copolyform modules. Commun. Algebra 30(3), 1461-1473 (2002).

[19] Wisbauer, R., Foundations of Module and Ring Theory, Gordon and Breach, Reading, 1991

[20] Wisbauer, R., Modules and Algebras: bimodule structure and group actions on algebras, Longman, Harlow, 1996

[21] Zelmanowitz, J., Representation of rings with faithful polyform modules, Commun. Algebra 14, 1141-1169 (1986)

[22] Zöschinger, H., Kosinguläre und kleine Moduln, Commun. Algebra, to appear

Departamento de Matemática Pura, Universidade do Porto, Portugal

Departamento de Matemática, Universidade Federal de Santa Catarina, Brasil 